\title{
An Adaptive Dynamic Surface Controller for Ultralow Altitude Airdrop Flight Path Angle with Actuator Input Nonlinearity
}

\author{
Mao-long Lv, Xiu-xia Sun, Shu-guang Liu, and Dong Wang \\ Aeronautics and Astronautics Engineering, Air Force Engineering University, Xi'an 710038, China \\ Correspondence should be addressed to Mao-long Lv; 18037707161@163.com
}

Received 23 March 2016; Accepted 20 June 2016

Academic Editor: Thierry Floquet

Copyright ( 2016 Mao-long Lv et al. This is an open access article distributed under the Creative Commons Attribution License, which permits unrestricted use, distribution, and reproduction in any medium, provided the original work is properly cited.

\begin{abstract}
In the process of ultralow altitude airdrop, many factors such as actuator input dead-zone, backlash, uncertain external atmospheric disturbance, and model unknown nonlinearity affect the precision of trajectory tracking. In response, a robust adaptive neural network dynamic surface controller is developed. As a result, the aircraft longitudinal dynamics with actuator input nonlinearity is derived; the unknown nonlinear model functions are approximated by means of the RBF neural network. Also, an adaption strategy is used to achieve robustness against model uncertainties. Finally, it has been proved that all the signals in the closed-loop system are bounded and the tracking error converges to a small residual set asymptotically. Simulation results demonstrate the perfect tracking performance and strong robustness of the proposed method, which is not only applicable to the actuator with input deadzone but also suitable for the backlash nonlinearity. At the same time, it can effectively overcome the effects of dead-zone and the atmospheric disturbance on the system and ensure the fast track of the desired flight path angle instruction, which overthrows the assumption that system functions must be known.
\end{abstract}

\section{Introduction}

Ultralow altitude airdrop (ULAA) is a crucial ability of a large transport aircraft, which is mainly applied in delivering heavyweight equipment to the precise desired region and critical to the success of military tasks $[1,2]$. The process of ultralow altitude airdrop includes five stages: preparation, falling, flat, tracking, and pull-up. Subsequent to the falling stage, heavyweight equipment and supplies drop to the desired location accurately. Uncertainty during the airdrop process is inevitable, so the model functions are very likely to be unknown. Besides, the ground effect $[3,4]$, sensor measurement error, the low altitude airflow [5], and other uncertain factors seriously disturb trajectory control and threaten flight safety and mission performance [6]. What is more, the aircraft with low-speed flying states demonstrates poor antiinterference performance, which is highly susceptible to low altitude atmospheric disturbances.

Over recent years, quite a few meaningful achievements have been made in developing advanced aircraft controllers to ensure the accuracy and aircraft safety of airdrop $[1,2,7]$. For example, it is proposed that a remarkable robustness of double ring mixed with iterative sliding-mode controller can reject constant uncertainties and uncertain atmospheric disturbances [1]. In addition, based on the decoupled and linearized aircraft model achieved by using the input-output feedback linearization approach, an iterative SM (slidingmode) flight controller is presented, which achieves a global dynamic switching function in the first level for the purpose of eliminating the reaching phase of the sliding motion. Meanwhile, a nonlinear function in the second level is designed to constitute an integral sliding manifold, which weakens the overcompensation of the integral term to big errors effectively [2]. Recently, a novel autopilot inner-loop based on LQR and $L_{1}$ adaptive approach that employs a semilinear time-varying system with cargo disturbances to approximate the model nonlinearities is presented to suppress the unknown disturbances caused by cargo movements [7]. However, it is worth noting that when designing the controller, the above references do not consider actuator input nonlinearities such as dead-zone and backlash and ignore the actuator dynamic characteristics and nonlinear factors; instead, they consider that the actual deflection angle is equal to the rudder angle instruction [8]. Nonetheless, 
because the actual steering control rudder deflection actuator includes mechanical link and hydraulic transmission device which inevitably lead to dead-zone or backlash nonlinearity in the steering gear, the stability of the system is undermined and even system divergence might occur as a result [9].

For the moment, controllers that consider actuator input dead-zone or backlash of transport have not been reported, but the control methods used in nonlinear system with deadzone or backlash have already been extensively researched [10-14]. For example, a novel adaptive fuzzy backstepping control method is developed, which uses fuzzy logic systems to approximate the unknown nonlinear functions and a fuzzy filter state observer to estimate the immeasurable states [10]. Recently, an adaptive fuzzy decentralized output feedback control scheme based on the adaptive backstepping DSC design technique has been proposed to be employed in a class of interconnected nonlinear pure-feedback systems [11]. Moreover, an adaptive fuzzy robust output feedback control problem is considered in a class of SISO nonlinear systems in a strict-feedback form, which first uses fuzzy logic systems to approximate the unstructured uncertainties and later utilizes the information of bounds of dead-zone slopes and treats the time-varying inputs coefficients as a system uncertainty [12]. What is more, as for a class of pure-feedback uncertain nonlinear systems with unknown dead-zone inputs and immeasurable states, based on the information of the dead-zone slopes and the unknown inputs coefficients that are treated as a system uncertainty, an adaptive fuzzy output feedback control method is proposed via the backstepping recursive design technique [13].

In the execution of the input nonlinearity of airdrop decline phase of flight path angle that tracks control problem, this paper proposes an adaptive neural network dynamic surface control method, which boasts a first-order lowpass filter introduced in the traditional backstepping control technique to avoid explosion of differential problems. The adaptive law is used to estimate the unknown model errors and external disturbance. Besides, the robust compensation term and neural network are introduced to implement the closed-loop system stability control, which effectively eliminates the adverse effect produced by actuator nonlinearity on the system. Moreover, it has been proven that the designed controller is able to guarantee that all signals are semiglobally uniformly ultimately bounded. Finally, simulation verifies the feasibility and effectiveness of the obtained theoretical results.

\section{Problem Statement}

2.1. Aircraft Modeling with Actuator Input Nonlinearity. During the airdrop decline stage, the pilot mainly uses frequent manipulation servo to drive the rudder deflection to ensure that the aircraft quickly and accurately tracks the reference flight path angle instruction. In this process, aircraft model that only considers longitudinal motion can be depicted as follows [1]:

$$
\begin{aligned}
& \dot{\gamma}=-f_{6}-f_{5} \gamma-f_{4} \cos \gamma+f_{5} \theta+\Delta d_{w}(C, \gamma, \theta) \\
& \dot{\theta}=q
\end{aligned}
$$

$$
\begin{aligned}
\dot{q}= & f_{0}+f_{1} q+f_{2} \theta-f_{2} \gamma+f_{3} \delta(u) \\
& +\Delta d_{n}(C, \gamma, \theta, q, \delta) \\
\delta(u)= & f_{\delta}(u),
\end{aligned}
$$

where $\gamma$ is the flight path angle; $\theta=\gamma+\alpha$ with $\theta$ being the pitch angle; $q$ is the pitch rate; $u$ is rudder angle instruction; and $\delta$ is the servo actuator driving actual rudder angle. $f_{\delta}(\cdot)$ is the servo actuator nonlinearities; $f_{0}=\bar{q} S c_{A} C_{m 0} / I_{y}, f_{1}=$ $\bar{q} S c_{A} C_{m q} / I_{y}, f_{2}=\bar{q} S c_{A} C_{m \alpha} / I_{y}, f_{3}=\bar{q} S c_{A} C_{m \delta_{e}} / I_{y}, f_{4}=$ $g / V$, and $c_{A}$ is the mean aerodynamic chord; $f_{5}=\left(\bar{q} S c_{L \alpha}+\right.$ $T) /(m V), f_{6}=-\bar{q} S c_{L 0} /(m V)$, and $S$ is the wing area; $I_{y}$ is the pitch moment of inertia; $m$ is the mass of the aircraft; $V$ is the airspeed; $T$ is the engine thrust, and $\bar{q}=\rho V^{2} / 2$ is the dynamic pressure; $\rho$ is the air mass density; $C_{m *}$ is the pitch moment coefficients and $C_{L^{*}}$ is the lift coefficients.

Assumption 1. There always exist uncertain functions, and $\Delta d_{w}(C, \gamma, \theta)$ and $\Delta d_{n}(C, \gamma, \theta, q, \delta)$ satisfy $\left|\Delta d_{w}(C, \gamma, \theta)\right| \leq$ $\Delta D_{w}$ and $\left|\Delta d_{n}\left(C, \gamma, \theta, q, \delta_{e}\right)\right| \leq \Delta D_{n}$, where $\Delta D_{w}>0$ and $\Delta D_{n}>0$ are unknown constants.

2.2. Actuator Dead-Zone or Backlash Nonlinearity Model. According to the actual aircraft actuator that performs with dead-zone and backlash, a class of nonlinearities can be represented by a generalized model as follows:

$$
f_{\delta}(u)=k(u, t) \cdot u+\varepsilon_{\delta}(u)
$$

where $k(u, t)>0$ is an unknown continuous function and $\varepsilon_{\delta}(u)$ is the bounded modeling error which satisfies $\left|\varepsilon_{\delta}(u)\right| \leq$ $\varepsilon_{\delta}^{*}$ with $\varepsilon_{\delta}^{*}$ being an unknown constant.

Assumption 2. The slope of nonlinearity characteristic $k(u, t)$ is bounded and there always exist unknown constants $k_{\min }>$ 0 and $k_{\max }>0$ satisfying $0<k_{\min } \leq k(u, t) \leq k_{\max }$.

Case 1. When considering the dead-zone nonlinearity, $\delta(u)$ can be described as

$$
\delta(u)= \begin{cases}k(u, t)\left(u-b_{r}\right), & u \geq b_{r} \\ 0, & -b_{l}<u<b_{r} \\ k(u, t)\left(u+b_{l}\right), & u \leq-b_{l},\end{cases}
$$

where $k(u, t)$ stands for the slope of the dead-zone characteristic, $b_{r}$ and $b_{l}$ represent the breakpoints of the dead-zone nonlinearity, $b_{r}$ and $b_{l}$ are unknown positive constants, and the $\varepsilon_{\delta}(u)$ function is chosen as

$$
\varepsilon_{\delta}(u)= \begin{cases}-k(u, t) b_{r}, & u \geq b_{r} \\ -k(u, t) u, & -b_{l}<u<b_{r} \\ k(u, t) b_{l}, & u \leq-b_{l} .\end{cases}
$$

According to (4) and Assumption 2, it can be inferred that $\left|\varepsilon_{\delta}(u)\right| \leq \varepsilon_{\delta}^{*}=k_{\max } \cdot \max \left\{\left|b_{r}\right|,\left|b_{l}\right|\right\}$. 
Case 2. When considering backlash nonlinearity, the analytical expression of $\delta(u)$ can be delivered as

$$
\begin{aligned}
& \delta(u) \\
& \quad= \begin{cases}k(u, t)\left(u-B_{r}\right), & \dot{u}>0, \delta=k(u, t)\left(u-B_{r}\right) \\
k(u, t)\left(u-B_{l}\right), & \dot{u}<0, \delta=k(u, t)\left(u-B_{l}\right) \\
\delta\left(t_{-}\right), & \text {others, }\end{cases}
\end{aligned}
$$

where $k(u, t)>0$ is the slope of backlash and $B_{r}>0$ and $B_{l}<0$ are relative positions and they are constant parameters. The function $\varepsilon_{\delta}(u)$ in model (2) is chosen as

$$
\varepsilon_{\delta}(u)= \begin{cases}-k(u, t) B_{r}, & \dot{u}>0, \quad \delta=k(u, t)\left(u-B_{r}\right) \\ -k(u, t) B_{l}, & \dot{u}<0, \quad \delta=k(u, t)\left(u-B_{l}\right) \\ \delta\left(t_{-}\right)-k u, & \text { others. }\end{cases}
$$

As a result, Assumption 2 is satisfied and $\left|\varepsilon_{\delta}(u)\right| \leq \varepsilon_{\delta}^{*}=k_{\max }$. $\max \left\{\left|b_{r}\right|,\left|b_{l}\right|\right\}$. Therefore, it can be seen from the above that dead-zone and backlash nonlinearities can be viewed as the particular cases of the input nonlinearity in our paper.

Assumption 3. The reference flight path angle instructions $y_{d}$, $\dot{y}_{d}$, and $\ddot{y}_{d}$ are smooth and bounded, and they are included in the compact set $\Omega_{1}$ as follows:

$$
\Omega_{1}=\left\{\left(\gamma_{d}, \dot{\gamma}_{d}, \ddot{\gamma}_{d}\right) \mid \gamma_{d}^{2}+\dot{\gamma}_{d}^{2}+\ddot{\gamma}_{d}^{2} \leq K_{0}\right\}
$$

where $K_{0}>0$ is a constant.

Control Objective. As for the aircraft longitudinal model with actuator nonlinearity, uncertain external atmospheric disturbance, unknown model function, and Nussbaum-gain technique will be used in this paper to design controller so that the flight path angle $y$ can track the reference flight path angle $y_{d}$ instruction quickly and accurately.

Remark 4. To facilitate the representation, define variables $\left[x_{1}, x_{2}, x_{3}\right]^{T}=[\gamma, \theta, q]^{T} ; \Delta d_{w}(\cdot), \Delta d_{n}(\cdot), \Delta D_{w}(\cdot)$, and $\Delta D_{n}(\cdot)$ are replaced by $\Delta d_{w}, \Delta d_{n}, \Delta D_{w}$, and $\Delta D_{n}$, and, as a result, the system model (1) can be rewritten as

$$
\begin{aligned}
\dot{x}_{1} & =f_{5} x_{2}+g_{1}\left(\bar{x}_{1}\right)+\Delta d_{w}, \\
\dot{x}_{2} & =x_{3}+g_{2}\left(\bar{x}_{2}\right), \\
\dot{x}_{3} & =f_{3} \delta(u)+g_{3}\left(\bar{x}_{3}\right)+\Delta d_{n}, \\
\delta(u) & =k(u, t) \cdot u+\varepsilon_{\delta}(u), \\
y & =x_{1},
\end{aligned}
$$

where $g_{1}\left(\bar{x}_{1}\right)=-f_{6}-f_{5} x_{1}-f_{4} \cos x_{1}, g_{2}\left(\bar{x}_{2}\right)=0$, and $g_{3}\left(\bar{x}_{3}\right)=f_{0}+f_{1} x_{3}+f_{2} x_{2}-f_{2} x_{1}$ are unknown smooth system functions. In this regard, $\bar{x}_{1}=x_{1}, \bar{x}_{2}=\left[\begin{array}{ll}x_{1} & x_{2}\end{array}\right]^{T}$, and $\bar{x}_{3}=\left[\begin{array}{lll}x_{1} & x_{2} & x_{3}\end{array}\right]^{T}$.
2.3. Nussbaum-Type Gain. Because the Nussbaum-gain technique is used in this paper, some results for Nussbaum-gain are presented as follows.

A function $N(\cdot)$ is called a Nussbaum-type function if it is even and smooth and possesses the following properties [15]:

$$
\begin{aligned}
& \lim _{p \rightarrow \infty} \sup \frac{1}{p} \int_{0}^{p} N(\zeta) d \zeta=+\infty, \\
& \lim _{p \rightarrow \infty} \inf \frac{1}{p} \int_{0}^{p} N(\zeta) d \zeta=-\infty .
\end{aligned}
$$

Lemma 5 (see [16]). Make $V(\cdot)$ and $\zeta(\cdot)$ smooth functions defined in $\left[0, t_{f}\right)$ with $V(t) \geq 0, \forall t \in\left[0, t_{f}\right)$, where $t_{f} \in$ $[0, \infty] . N(\zeta)$ is an even and smooth Nussbaum function. If the following inequality holds

$$
\begin{array}{r}
V(t) \leq \kappa_{1}+e^{-\kappa_{2} t} \int_{0}^{t}[g(x(\tau)) N(\zeta(\tau))+1] \dot{\zeta} e^{\kappa_{2} \tau} d \tau \\
\forall t \in\left[0, t_{f}\right),
\end{array}
$$

where $\kappa_{1}$ represents a suitable constant, $\kappa_{2}$ is a positive constant, and $g(x(\tau))$ is a time-varying parameter, which takes value in the unknown closed intervals $I=\left[l^{-}, l^{+}\right]$, with $0 \notin I$, then $V(t)$, $\zeta(t)$, and $\int_{0}^{t} N(\zeta(\tau)) \dot{\zeta} d \tau$ must be bounded in $\left[0, t_{f}\right)$.

Lemma 6 (see [17]). The hyperbolic tangent function $\tanh (\cdot)$ will be used in this paper, and it is commonly believed that it is continuous, differentiable, and monotonic, and it satisfies that for any $v>0$ and $q \in R$

$$
\begin{aligned}
& 0 \leq|q|-q \tanh \left(\frac{q}{v}\right) \leq 0.2785 v, \\
& 0 \leq q \tanh \left(\frac{q}{v}\right)
\end{aligned}
$$

Remark 7. Throughout this paper, make $\|\cdot\|$ denote the 2norm, $\hat{\cdot}$ is the estimate of.$^{*}$, the estimate error is $\widetilde{\cdot}=\cdot^{*}-\hat{\bullet}$, and $\lambda_{\max }(A)$ denotes the largest eigenvalue of a square matrix A.

\section{Adaptive Flight Controller Design}

3.1. NN Basics. Considering the unknown nonlinear function of model (8), this paper uses the RBF neural network to approximate the unknown function $g_{i}\left(\bar{x}_{i}\right)$ :

$$
g_{i}\left(\bar{x}_{i}\right)=W_{i}^{* T} \Phi_{i}\left(\bar{x}_{i}\right)+\varepsilon_{i}\left(\bar{x}_{i}\right)
$$

where $W_{i}^{*}=\arg \min _{\widehat{W}_{i}^{T} \in R^{s_{i}}}\left\{\sup _{\bar{x}_{i} \in R^{i}}\left\|g_{i}\left(\bar{x}_{i}\right)-\widehat{W}_{i}^{T} \Phi_{i}\left(\bar{x}_{i}\right)\right\|\right\}$ is the optimal weight vector and is an unknown constant parameter, $\Phi_{i}\left(\bar{x}_{i}\right): R^{i} \rightarrow R^{s_{i}}$ is the vector of the basis function, and $s_{i}$ is the number of nodes in the neural network i. $\widehat{W}_{i}$ is the estimate of $W_{i}^{*}$, and define the estimate error as $\widetilde{W}_{i}=W_{i}^{*}-\widehat{W}_{i} . \Phi_{i}$ and $\varepsilon_{i}$ indicate $\Phi_{i}\left(\bar{x}_{i}\right)$ and $\varepsilon_{i}\left(\bar{x}_{i}\right)$, respectively, $\varepsilon_{i}$ is neural network approximation error that satisfies $\left|\varepsilon_{i}\right| \leq \varepsilon_{i}^{*}$, and $\varepsilon_{i}^{*}$ is an unknown positive constant. 
3.2. Controller Design. Based on the backstepping progressive controller design method, the adaptive law is introduced to estimate the unknown parameters of the system, and the design steps of the adaptive dynamic surface controller are as follows.

To begin with, define the first tracking error as $e_{1}=x_{1}-$ $\gamma_{d}$, and the time derivative of $e_{1}$ is

$$
\dot{e}_{1}=f_{5} x_{2}+g_{1}\left(\bar{x}_{1}\right)+\Delta d_{w}-\dot{\gamma}_{d} \text {. }
$$

Combining (11), we can rewrite (13) as

$$
\dot{e}_{1}=f_{5} x_{2}+W_{1}^{* T} \Phi_{1}+\varepsilon_{1}+\Delta d_{w}-\dot{\gamma}_{d}
$$

The virtual control law and adaptive law of parameters are designed as follows:

$$
\begin{aligned}
\alpha_{1} & =-\frac{1}{f_{5}}\left(k_{1} e_{1}+\widehat{W}_{1}^{T} \Phi_{1}+\widehat{\delta}_{1} \tanh \left(\frac{e_{1}}{v_{1}}\right)-\dot{\gamma}_{d}\right), \\
\dot{\widehat{W}}_{1} & =\Gamma_{1}\left(\Phi_{1} e_{1}-\sigma_{1} \widehat{W}_{1}\right), \\
\dot{\hat{\delta}}_{1} & =\mu_{1}\left(e_{1} \tanh \left(\frac{e_{1}}{v_{1}}\right)-\sigma_{2} \widehat{\delta}_{1}\right),
\end{aligned}
$$

where $\delta_{1}^{*}=\varepsilon_{1}^{*}+\Delta D_{w}, \widehat{W}_{1}$ and $\widehat{\delta}_{1}$ are the estimates of $W_{1}^{*}$ and $\delta_{1}^{*}$, respectively, and $k_{1}>0, v_{1}>0, \mu_{1}>0, \sigma_{1}>0, \sigma_{2}>0$ are design parameters. $\Gamma_{1}=\Gamma_{1}^{-1}$ is the adaptive gain matrix. The term $\dot{\widehat{\delta}}_{1}$ in (16) is viewed as a robust compensator which can reject the influence of modeling approximation error and external disturbance.

To avoid repeatedly differentiating $\alpha_{1}$ which results in the "explosion of complexity," make $\alpha_{1}$ pass through a first-order filter with the time constant $\tau_{2}>0$ to acquire $\alpha_{2, f}$ as

$$
\begin{aligned}
\tau_{2} \dot{\alpha}_{2, f}(t)+\alpha_{2, f}(t) & =\alpha_{1}(t), \\
\alpha_{2, f}(0) & =\alpha_{1}(0) .
\end{aligned}
$$

Subsequently, define the second tracking error variable as $e_{2}=x_{2}-\alpha_{2, f}$, and the time derivative of $e_{2}$ is

$$
\dot{e}_{2}=x_{3}-\dot{\alpha}_{2, f} \text {. }
$$

Similarly, design the virtual law and the parameter adaptive law as follows:

$$
\begin{aligned}
\alpha_{2} & =-\left(k_{2} e_{2}+\widehat{W}_{2}^{T} \Phi_{2}+\widehat{\delta}_{2} \tanh \left(\frac{e_{2}}{v_{2}}\right)-\dot{\alpha}_{2, f}\right) \\
\dot{\widehat{W}}_{2} & =\Gamma_{2}\left(e_{2} \Phi_{2}-\sigma_{3} \widehat{W}_{2}\right) \\
\dot{\widehat{\delta}}_{2} & =\mu_{2}\left(e_{2} \tanh \left(\frac{e_{2}}{v_{2}}\right)-\sigma_{4} \widehat{\delta}_{2}\right)
\end{aligned}
$$

where $\delta_{2}^{*}=\varepsilon_{2}^{*}, \widehat{W}_{2}$ and $\widehat{\delta}_{2}$ are the estimates of $W_{2}^{*}$ and $\delta_{2}^{*}$, respectively, and $k_{2}>0, v_{2}>0, \mu_{2}>0, \sigma_{3}>0$, and $\sigma_{4}>0$ are design parameters. $\Gamma_{2}=\Gamma_{2}^{-1}$ is the adaptive gain matrix.
Likewise, make $\alpha_{2}$ pass through a first-order filter with time constant $\tau_{3}>0$ to achieve $\alpha_{3, f}$ as

$$
\begin{array}{r}
\tau_{3} \dot{\alpha}_{3, f}(t)+\alpha_{3, f}(t)=\alpha_{2}(t) \\
\alpha_{3, f}(0)=\alpha_{2}(0)
\end{array}
$$

Design the third tracking error variable $e_{3}=x_{3}-\alpha_{3, f}$, note (8) and (12), and the time derivative of $e_{3}$ is

$$
\begin{aligned}
\dot{e}_{3}= & f_{3}\left(k(u, t) \cdot u+\varepsilon_{\delta}(u)\right)+W_{3}^{* T} \Phi_{3}+\varepsilon_{3}+\Delta d_{n} \\
& -\dot{\alpha}_{3, f} .
\end{aligned}
$$

Finally, the virtual control law and adaptive law of parameters are designed as follows:

$$
\begin{aligned}
u & =N(\zeta)\left[k_{3} e_{3}+\widehat{W}_{3}^{T} \Phi_{3}+\widehat{\delta}_{3} \tanh \left(\frac{e_{3}}{v_{3}}\right)-\dot{\alpha}_{3, f}\right], \\
\dot{\zeta} & =k_{3} e_{3}^{2}+e_{3} \widehat{W}_{3}^{T} \Phi_{3}+\widehat{\delta}_{3} e_{3} \tanh \left(\frac{e_{3}}{v_{3}}\right)-e_{3} \dot{\alpha}_{3, f}, \\
\dot{\widehat{W}}_{3} & =\Gamma_{3}\left(e_{3} \Phi_{3}-\sigma_{5} \widehat{W}_{3}\right), \\
\dot{\widehat{\delta}}_{3} & =\mu_{3}\left(e_{3} \tanh \left(\frac{e_{3}}{v_{3}}\right)-\sigma_{6} \widehat{\delta}_{3}\right),
\end{aligned}
$$

where $\delta_{3}^{*}=\left|f_{3}\right| \varepsilon_{\delta}^{*}+\varepsilon_{3}^{*}+\Delta D_{n}, k_{3}>0, v_{3}>0, \mu_{3}>0, \sigma_{5}>0$, and $\sigma_{6}>0$ are design parameters, $\widehat{W}_{3}$ and $\widehat{\delta}_{3}$ are the estimates of $W_{3}^{*}$ and $\delta_{3}^{*}$, respectively, and $\Gamma_{3}=\Gamma_{3}^{-1}$ is the adaptive gain matrix.

\section{Stability and Tracking Performance Analysis of the Controller}

Theorem 8. According to the control system (8), for the closedloop system composed of control law (14), (19), (23), and (24) and the adaptive law of parameters (15), (20), and (25), if Assumptions 1 3 are satisfied and the initial states of system are bounded, control parameters $\sigma_{i}(i=1, \ldots, 6), k_{i}, v_{i}$, and $\tau_{i}(i=2,3)$ will make all variables of the closed-loop system semiglobally uniformly ultimately bounded with tracking error converging to zero.

Define the third-order subsystem Lyapunov function $V_{3}$ :

$$
V_{3}=\frac{1}{2} e_{3}^{2}+\frac{1}{2} \widetilde{W}_{3}^{T} \Gamma_{3}^{-1} \widetilde{W}_{3}+\frac{1}{2 \mu_{3}} \widetilde{\delta}_{3}^{2} .
$$

Combining (22), (23), and (25), the time derivative of (26) is

$$
\begin{aligned}
\dot{V}_{3} \leq & f_{3} k(u, t) N(\zeta) \dot{\zeta}+\left|e_{3}\right|\left(\left|f_{3}\right| \varepsilon_{\delta}^{*}+\varepsilon_{3}^{*}+\Delta D_{n}\right) \\
& +\frac{\left(e_{3} y_{3}\right)}{\tau_{3}}+e_{3} W_{3}^{* T} \Phi_{3}-\widetilde{W}_{3}^{T} \Gamma_{3}^{-1} \dot{\widehat{W}}_{3}-\frac{1}{\mu_{3}} \widetilde{\delta}_{3} \dot{\widehat{\delta}}_{3} .
\end{aligned}
$$


Adding and subtracting $\dot{\zeta}$ on the right-hand side of (27), one has

$$
\begin{aligned}
\dot{V}_{3} \leq & {\left[f_{3} k(u, t) N(\zeta)+1\right] \dot{\zeta} } \\
& +\delta_{3}^{*}\left(\left|e_{3}\right|-e_{3} \tanh \left(\frac{e_{3}}{v_{3}}\right)\right) \\
& -\widetilde{W}_{3}^{T} \Gamma_{3}^{-1}\left(\dot{\widehat{W}}_{3}-e_{3} \Gamma_{3} \Phi_{3}\right) \\
& -\frac{\widetilde{\delta}_{3}}{\mu_{3}}\left(\dot{\widehat{\delta}}_{3}-e_{3} \mu_{3} \tanh \left(\frac{e_{3}}{v_{3}}\right)\right)-k_{3} e_{3}^{2} .
\end{aligned}
$$

Substitute (28) for (25) and the application of Lemma 6 leads to

$$
\begin{aligned}
\dot{V}_{3} \leq & {\left[f_{3} k(u, t) N(\zeta)+1\right] \dot{\zeta}+0.2785 \delta_{3}^{*} v_{3}-k_{3} e_{3}^{2} } \\
& +\sigma_{5} \widetilde{W}_{3}^{T} \widehat{W}_{3}+\sigma_{6} \widetilde{\delta}_{3} \widehat{\delta}_{3} .
\end{aligned}
$$

Use the following inequalities:

$$
\begin{aligned}
& \sigma_{5} \widetilde{W}_{3}^{T} \widehat{W}_{3} \leq-\frac{\sigma_{5}}{2}\left\|\widetilde{W}_{3}\right\|^{2}+\frac{\sigma_{5}}{2}\left\|W_{3}^{*}\right\|^{2}, \\
& \sigma_{6} \widetilde{\delta}_{3} \widehat{\delta}_{3} \leq-\frac{\sigma_{6}}{2} \widetilde{\delta}_{3}^{2}+\frac{\sigma_{6}}{2} \delta_{3}^{* 2} .
\end{aligned}
$$

Noting (29), one can obtain

$$
\dot{V}_{3} \leq-\beta V+\left[f_{3} k(u, t) N(\zeta)+1\right] \dot{\zeta}_{3}+a_{0}
$$

where $\beta=\min \left\{2 k_{3}, \mu_{3} \sigma_{6}, \sigma_{5} / \lambda_{\max }\left(\Gamma_{3}^{-1}\right)\right\}$ and $a_{0}=$ $0.2785 \delta_{3}^{*} v_{3}+\left(\sigma_{6} / 2\right) \delta_{3}^{* 2}+\left(\sigma_{5} / 2\right)\left\|W_{3}^{*}\right\|^{2}$.

Multiply (31) by $e^{\beta t}$, and then integrate (31) in $[0, t]$. Thus,

$$
\begin{aligned}
\dot{V}_{3} \leq & \int_{0}^{t}\left[f_{3} k(u, \tau) N(\zeta)+1\right] \dot{\zeta} e^{-\beta(t-\tau)} d \tau+\frac{a_{0}}{\beta} \\
& +V_{3}(0) .
\end{aligned}
$$

According to Assumption 2 and Lemma 5, $V_{3}(t), \zeta(t)$, and the term $\int_{0}^{t} f_{3} k(u, \tau) N(\zeta) \dot{\zeta} d \tau$ are bounded.

Define the upper bound $Q$ as follows:

$$
\int_{0}^{t}\left|f_{3} k(u, \tau) N(\zeta)+1\right| \dot{\zeta} e^{-\beta(t-\tau)} d \tau \leq Q .
$$

From (32) and (33), we can get

$$
V_{3} \leq \frac{a_{0}}{\beta}+V_{3}(0)+Q
$$

Notice (26) and (34), $V_{3}(t)$ is bounded, and

$$
\begin{aligned}
& \left|e_{3}\right| \leq \sqrt{2 V_{3}(t)} \leq \sqrt{2\left(\frac{a_{0}}{\beta}+V_{3}(0)+Q\right)}=M, \\
& \left|\widetilde{\delta}_{3}\right| \leq \sqrt{2 V_{3}(t)}=M, \\
& \left|\widetilde{W}_{3}\right| \leq \sqrt{2 V_{3}(t)}=M,
\end{aligned}
$$

where $Q>0$ and $M>0$ are unknown constants.
Define the output errors $y_{2}=\alpha_{2, f}-\alpha_{1}$ and $y_{3}=\alpha_{3, f}-\alpha_{2}$, and from $(13) \sim(16),(18) \sim(21)$, and $(23) \sim(26)$ we can know that there exist continuous functions $B_{2}(\cdot)$ and $B_{3}(\cdot)$ that satisfy

$$
\begin{aligned}
& \left|\dot{y}_{2}+\frac{y_{2}}{\tau_{2}}\right| \leq B_{2}\left(e_{1}, e_{2}, y_{2}, \widetilde{W}_{1}, \widetilde{\delta}_{1}, \gamma_{d}, \dot{\gamma}_{d}, \ddot{\gamma}_{d}\right), \\
& \left|\dot{y}_{3}+\frac{y_{3}}{\tau_{3}}\right| \\
& \quad \leq B_{3}\left(e_{1}, e_{2}, e_{3}, \widetilde{W}_{1}, \widetilde{W}_{2}, \widetilde{\delta}_{1}, \widetilde{\delta}_{2}, y_{2}, y_{3}, \gamma_{d}, \dot{\gamma}_{d}, \ddot{\gamma}_{d}\right) .
\end{aligned}
$$

From (36), we can obtain the following inequalities:

$$
\begin{aligned}
& y_{2} \dot{y}_{2} \leq\left|y_{2}\right| B_{2}-\frac{y_{2}^{2}}{\tau_{2}}, \\
& y_{3} \dot{y}_{3} \leq\left|y_{3}\right| B_{3}-\frac{y_{3}^{2}}{\tau_{3}} .
\end{aligned}
$$

Identical to (26), define the first-order subsystem Lyapunov function:

$$
V_{1}=\frac{1}{2} e_{1}^{2}+\frac{1}{2} \widetilde{W}_{1}^{T} \Gamma_{1}^{-1} \widetilde{W}_{1}+\frac{1}{2 \mu_{1}} \widetilde{\delta}_{1}^{2}+\frac{1}{2} y_{2}^{2}
$$

Noticing $x_{2}=e_{2}+\alpha_{1}+y_{2}$, according to Lemma 6, (15), (16), and Young's inequalities $f_{5} e_{1} e_{2} \leq\left(f_{5}^{2} e_{1}^{2}\right) / 2+e_{2}^{2} / 2$ and $f_{5} e_{1} y_{2} \leq\left(f_{5}^{2} e_{1}^{2}\right) / 2+y_{2}^{2} / 2$, the time derivative of $V_{1}$ is

$$
\begin{aligned}
\dot{V}_{1} \leq & \left(f_{5}^{2}-k_{1}\right) e_{1}^{2}+\frac{e_{2}^{2}}{2}-\frac{\sigma_{1}}{2}\left\|\widetilde{W}_{1}\right\|^{2}-\frac{\sigma_{2}}{2} \widetilde{\delta}_{1}^{2}+\frac{1}{2} y_{2}^{2} \\
& +y_{2} \dot{y}_{2}+a_{1},
\end{aligned}
$$

where $a_{1}=\left(\sigma_{2} / 2\right) \delta_{1}^{* 2}+\left(\sigma_{1} / 2\right)\left\|W_{1}^{*}\right\|^{2}+0.2785 v_{1} \delta_{1}^{*}$. Subsequently, define the second-order subsystem Lyapunov function:

$$
V_{2}=\frac{1}{2} e_{2}^{2}+\frac{1}{2} \widetilde{W}_{2}^{T} \Gamma_{2}^{-1} \widetilde{W}_{2}+\frac{1}{2 \mu_{2}} \widetilde{\delta}_{2}^{2}+\frac{1}{2} y_{3}^{2} .
$$

By virtue of Lemma 6, the time derivative of $V_{2}$ is

$$
\begin{aligned}
\dot{V}_{2} \leq & \left(1-k_{2}\right) e_{2}^{2}+\frac{e_{3}^{2}}{2}-\frac{\sigma_{3}}{2}\left\|\widetilde{W}_{2}\right\|^{2}-\frac{\sigma_{4}}{2} \widetilde{\delta}_{2}^{2}+\frac{1}{2} y_{3}^{2} \\
& +y_{3} \dot{y}_{3}+a_{2},
\end{aligned}
$$

where $a_{2}=\sigma_{4} \delta_{2}^{* 2} / 2+\sigma_{3}\left\|W_{2}^{*}\right\|^{2} / 2+0.2785 v_{2} \delta_{2}^{*}$. According to (34) and (35), $V_{3}$ is bounded and $e_{3}, \widehat{W}_{3}$, and $\widehat{\delta}_{3}$ are semiglobally, uniformly, and ultimately stable and bounded.

In summary, define the following Lyapunov function:

$$
V=V_{1}+V_{2}
$$




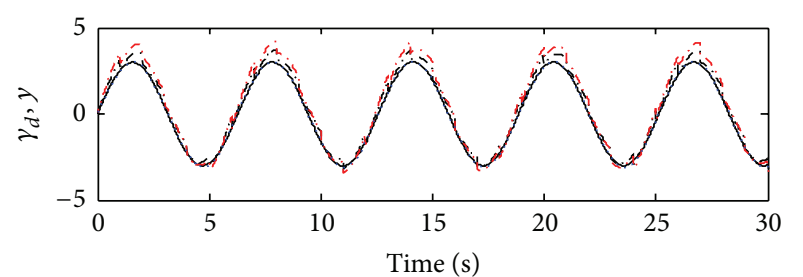

$-\mathrm{a}$

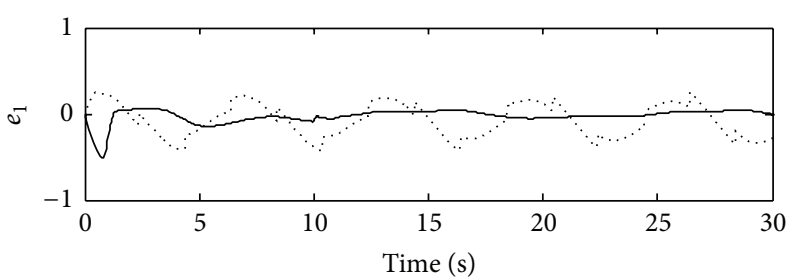

- Scheme 1

Scheme 2

FIGURE 1: Flight path angle and the track angle tracking error curves comparison.

Noting (37), (39), and (41), the time derivative of $V$ is

$$
\begin{aligned}
\dot{V} \leq & \left(f_{5}^{2}-k_{1}\right) e_{1}^{2}+\left(\frac{3}{2}-k_{2}\right) e_{2}^{2}-\frac{\sigma_{2} \widetilde{\delta}_{1}^{2}}{2}-\frac{\sigma_{4} \widetilde{\delta}_{2}^{2}}{2} \\
& -\frac{\sigma_{1}}{2}\left\|\widetilde{W}_{1}\right\|^{2}-\frac{\sigma_{3}}{2}\left\|\widetilde{W}_{2}\right\|^{2}+\sum_{i=2}^{3}\left(\left|y_{i}\right| B_{i}\right) \\
& +\sum_{i=2}^{3}\left[\left(-\frac{1}{\tau_{i}}+\frac{1}{2}\right) y_{i}^{2}\right]+a_{3},
\end{aligned}
$$

where $a_{3}=a_{1}+a_{2}+M^{2} / 2$, define the following set $\Omega_{2}=$ $\left\{\left(e_{1}, e_{2}, e_{3}, y_{2}, y_{3}, \widetilde{W}_{1}, \widetilde{W}_{2}, \widetilde{\delta}_{1}, \widetilde{\delta}_{2}\right)|V \leq P,| e_{3} \mid \leq M\right\}$, and $P>$ 0 is a constant, since $\Omega_{1}$ is a compact set. $\Omega_{1} \times \Omega_{2}$ is also compact, and it is easy to see from (36) and (37) that all the variables of the continuous functions $B_{2}(\cdot)$ and $B_{3}(\cdot)$ are in the set $\Omega_{1} \times \Omega_{2}$. Therefore, $B_{2}(\cdot)$ and $B_{3}(\cdot)$ have maximal $D_{2}(\cdot)$ and $D_{3}(\cdot)$ on $\Omega_{1} \times \Omega_{2}$, respectively. And then use the inequalities $\left|y_{2}\right|\left|D_{2}\right| \leq y_{2}^{2} D_{2}^{2} /\left(2 c_{2}\right)+c_{2} / 2,\left|y_{3}\right|\left|D_{3}\right| \leq y_{3}^{2} D_{3}^{2} /\left(2 c_{3}\right)+c_{3} / 2$, where $c_{2}>0$ and $c_{3}>0$ are design parameters. As a result, (43) can be written as

$$
\begin{aligned}
\dot{V} \leq & -\frac{\sigma_{1}}{2}\left\|\widetilde{W}_{1}\right\|^{2}-\frac{\sigma_{3}}{2}\left\|\widetilde{W}_{2}\right\|^{2} \\
& +\sum_{i=2}^{3}\left[\left(\frac{D_{i}^{2}}{\left(2 c_{i}\right)}-\frac{1}{\tau_{i}}+\frac{1}{2}\right) y_{i}^{2}\right]+\left(f_{5}^{2}-k_{1}\right) e_{1}^{2} \\
& +\left(\frac{3}{2}-k_{2}\right) e_{2}^{2}-\frac{\sigma_{2}}{2} \widetilde{\delta}_{1}^{2}-\frac{\sigma_{4}}{2} \widetilde{\delta}_{2}^{2}+a_{4},
\end{aligned}
$$

where $a_{4}=a_{3}+\left(c_{2}+c_{3}\right) / 2$, and, then, select $1 / \tau_{i}>D_{i}^{2} /\left(2 c_{i}\right)+$ $1 / 2+\lambda(i=2,3), k_{1}>f_{5}^{2}+k_{0}, k_{2}>3 / 2+k_{0}$, where $k_{0}>$ $0, \lambda>0$ are design parameters, we arrive at

$$
\dot{V} \leq-2 \mu V+a_{4} .
$$

Solving inequality (45), we can achieve $V \leq a_{4} /(2 \mu)+[V(0)-$ $\left.a_{4} /(2 \mu)\right] e^{-2 u t}$. Obviously, all the signals of the closed-loop system are bounded and we can get

$$
\lim _{t \rightarrow \infty} V(t) \leq \frac{a_{4}}{2 \mu}
$$

By increasing the design parameters $k_{0}, \lambda, \mu_{i}$, and $\sigma_{i}(i=$ $1, \ldots, 6)$ and meanwhile reducing $\lambda_{\max }\left(\Gamma_{1}^{-1}\right)$ and $\lambda_{\max }\left(\Gamma_{2}^{-1}\right)$, we can obtain $\mu>a_{4} /(2 P)$. When $V \geq P, \dot{V}<0, V \leq P$ is an invariant set. If $V(0) \leq P$, then $V(t) \leq P \forall t>0$. The tracking error can converge to a sphere with a radius of $a_{4} /(2 \mu)$. Choose $\mu>\max \left\{a_{4} /(2 P), a_{4} / \mathcal{E}\right\}$, and thus $e_{1}^{2} \leq 2 V \leq$ $\varepsilon$. By adjusting the design parameters, $\varepsilon$ can be arbitrarily small, and the tracking error can converge to any small area of the origin.

\section{Simulation Analysis}

An adaptive dynamic surface control law is designed to guarantee that the aircraft flight path angle $\gamma$ can track the desired trajectory $\gamma_{d}=3^{\circ} \sin (t)$ accurately. Assuming the atmospheric disturbances $\Delta d_{w}=0.02 \sin (2 t)$ and $\Delta d_{n}=$ $0.05 \cos (t)$, the initial states of the system are $x_{1}(0)=x_{2}(0)=$ $x_{3}(0)=0$ and the estimation initial values of adaptive parameters are set as $\widehat{\delta}_{1}(0)=\widehat{\delta}_{2}(0)=\widehat{\delta}_{3}(0)=0$ and $\zeta(0)=1$. Adaptive gain matrix is $\Gamma_{1}=\Gamma_{3}=\operatorname{diag}\{0.5\}$, the controller design parameters are determined as $\sigma_{1}=\sigma_{2}=\sigma_{5}=\sigma_{6}=$ $0.5, k_{0}=3, k_{1}=2.5, k_{2}=1.5, k_{3}=3.5, \tau_{1}=\tau_{2}=\tau_{3}=0.2$, and $c_{2}=c_{3}=1$, and $\lambda=0.5$ after experimental tuning, in which the Nussbaum function $N(\zeta)=e^{\zeta^{2}} \cos (\pi \zeta / 2)$ is used. Gauss function is selected as the basis function of radial basis neural network; as a result,

$$
\Phi(\mathbf{x})=e^{-\left(\mathbf{x}-\mu_{i}\right)^{T}\left(\mathbf{x}-\mu_{i}\right) / v_{i}^{2}}, \quad i=1,2, \ldots, l,
$$

where $\widehat{W}^{T} \Phi(\mathbf{x})$ contains $l=27$ nodes with centers evenly spaced in $[-4,4] \times[-4,4] \times[-4,4]$ and width $v_{i}=2$ and the initial values of the neural network weights $\widehat{W}(0)$ are set as 0 .

5.1. Control Performance Analysis with Considering DeadZone Nonlinearity. In order to investigate the influence of the dead-zone on airdrop control performance, the scheme proposed in this paper (scheme 1) is compared with the adaptive dynamic surface controller without considering actuator input dead-zone nonlinearity (scheme 2). The simulation results are presented in Figure 1.

First of all, the present study adopts scheme 2 to merely investigate the effect of dead-zone on closed-loop system without taking external disturbances into account. The deadzone model is shown in (48); simulation result is depicted as line $\mathrm{b}$ of Figure 1 . And line $\mathrm{a}$ is the desired flight path angle instruction. Drawing a comparison between line a and 

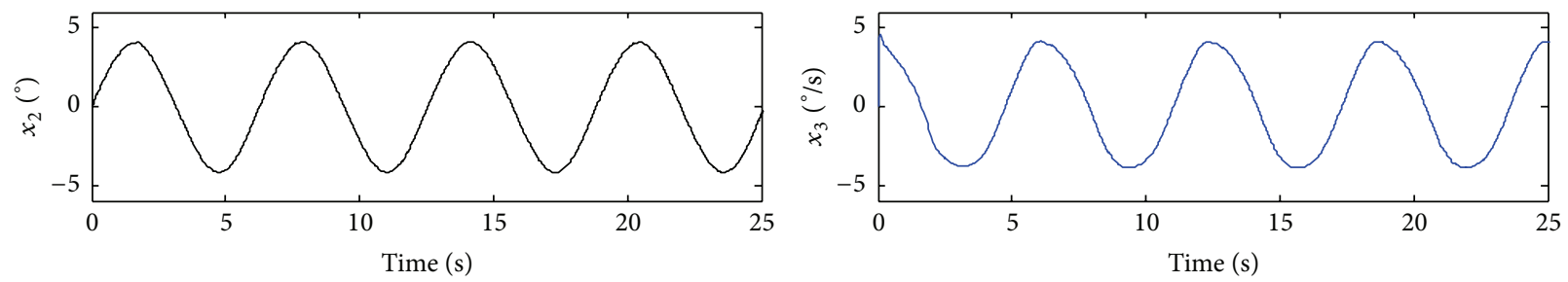

FIgURE 2: The curves of pitch angle and pitch rate.

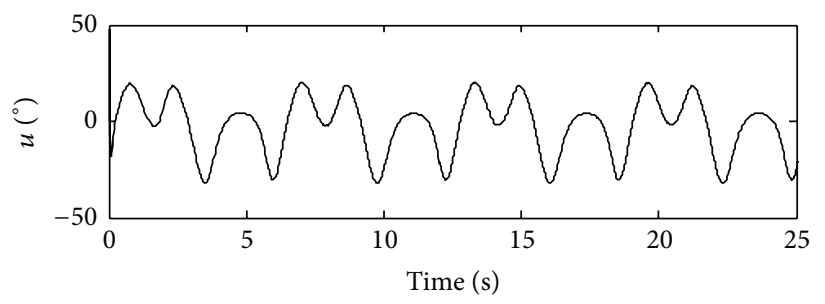

— Scheme 1

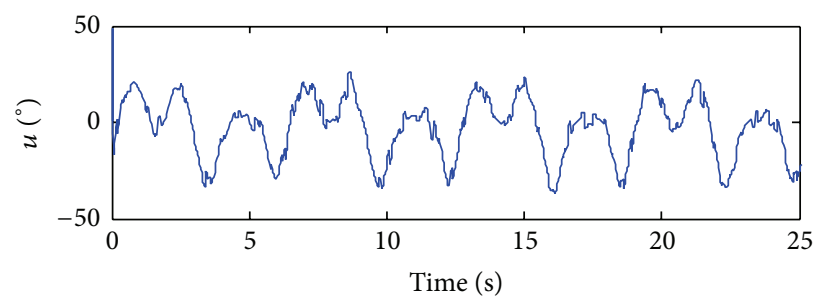

- Scheme 2

FIgURE 3: Comparison of control input curves.

line $b$, it can be easily seen that the dead-zone leads to the reduction of the performance of the control system, which renders the aircraft unable to track the desired trajectory command accurately.

Based on line $b$ coupled with the effects of outside atmosphere disturbance of $\Delta d_{w}$ and $\Delta d_{n}$ on the aircraft control performance, the simulation result is shown as line $\mathrm{c}$ in Figure 1. It can be seen that the aircraft flight path angle tracking performance declines remarkably, which aggravates the instability of the closed-loop system and undermines the accuracy and safety of airdrop.

\subsection{Tracking Control Analysis with Considering \\ Actuator Input Nonlinearity}

Example 9. When dead-zone nonlinearity happens to be present in system (8), choose the expression of $\delta(u)$ as follows:

$$
\delta(u)= \begin{cases}1.2(u-0.35), & u \geq 0.35 \\ 0, & -0.35<u<0.35 \\ 1.2(u+0.35), & u \leq-0.35 .\end{cases}
$$

The initial conditions and atmosphere disturbance expressions remain unchanged, simulation results are shown as line $\mathrm{d}$ and line $\mathrm{c}$ of Figure 1, as well as in Figures 2 and 3, where line $\mathrm{d}$ is the tracking curve of flight path angle with scheme 1 .

It can be seen from Figure 1 that the flight controller (scheme 1) can effectively overcome the effects of dead-zone and the atmospheric disturbance on the system, ensure the fast track of the desired flight path angle instruction, and track rapid error that converges to zero. Compared with the scheme proposed in this paper, there are palpably much more tracking errors in scheme 2. It can be easily seen from Figure 2 that, by virtue of the method of this paper, the trend of system state variables turns stable. From Figure 3, it can be seen that scheme 1 can effectively overcome the problem of control input flutter caused by dead-zone nonlinearity.

Example 10. When the backlash nonlinearity is concerned, choose the expression of $\delta(u)$ as follows:

$$
\begin{aligned}
& \delta(u) \\
& = \begin{cases}1.2\left(u-\frac{1}{57.3}\right), & \dot{u}>0, \delta=1.2\left(u-\frac{1}{57.3}\right), \\
1.2\left(u+\frac{1}{57.3}\right), & \dot{u}<0, \delta=1.2\left(u+\frac{1}{57.3}\right), \\
\delta\left(t_{-}\right), & \text {others. }\end{cases}
\end{aligned}
$$

The controller is identical to that of Example 9 without changing the control parameters, initial conditions, and Nussbaum functions. Simulation results are as demonstrated in Figures 4 and 5.

From Figure 4, we can infer that when considering actuator input backlash nonlinearity, scheme 1 can achieve tracking control performance as good as that of dead-zone nonlinearity; it effectively overcomes the adverse effects of backlash nonlinearity on the system and boasts considerable robustness. According to Figure 5, the estimation of the unknown parameters values gradually approaches the actual values with satisfactory and fast approximation.

\section{Conclusions}

The method proposed in this paper boasts the following advantages. Firstly, the approach can accurately estimate the unknown model parameters and use the neural networks to approximate the unknown system functions. In this way, the assumption that model function must be identified was overthrown. Secondly, this scheme introduces a robust 


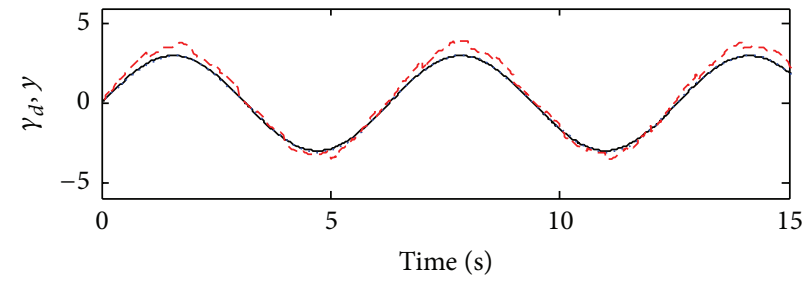

- Desired instruction $\quad---$ Scheme 2 Scheme 1

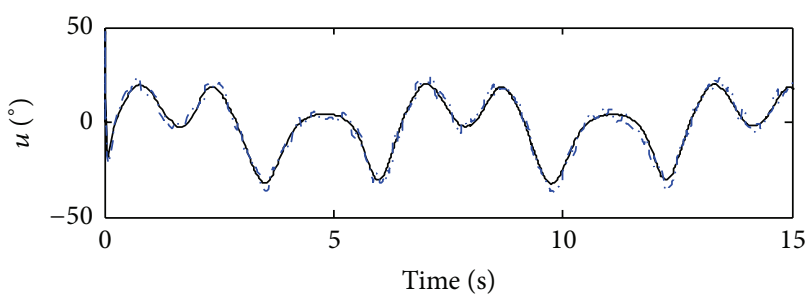

- Scheme 1

-..- Scheme 2

FIGURE 4: Comparison of flight path angle and control input curves.
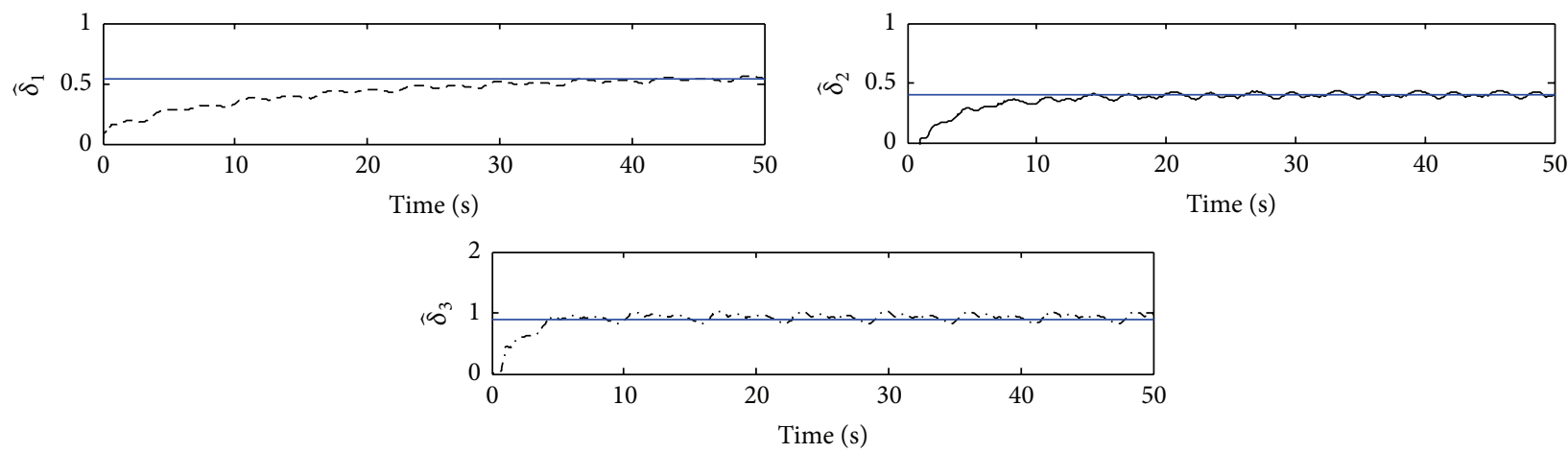

FIGURE 5: The curves of adaptive parameter estimation.

adaptive compensation term, which effectively eliminates the adverse effects of external atmospheric disturbances, the neural network approximation error, and actuator nonlinear modeling error on the system. Finally, the approach has a reference value to a certain extent for solving the tracking control problem with a class of uncertain nonlinear systems with input nonlinearity, which is similar to the structure examined in the present study.

\section{Competing Interests}

The authors declare that there is no conflict of interests regarding the publication of this paper.

\section{Acknowledgments}

This work is supported by the China Postdoctoral Science Foundation (Grant no. 2014M562629) and the Aviation Science Foundation of China (Grant no. 20135896025; Grant no. 20141396012; Grant no. 20155896025).

\section{References}

[1] R. Liu, X. X. Sun, and W. H. Dong, "Hybrid iteration sliding mode control for ultra-low altitude airdrop level off," Journal of Beijing University of Aeronautics and Astronautics, vol. 41, no. 1, pp. 83-89, 2015 (Chinese).

[2] R. Liu, X. X. Sun, and W. H. Dong, "Dynamics modeling and control of a transport aircraft for ultra-low altitude airdrop," Chinese Journal of Aeronautics, vol. 28, no. 2, pp. 478-487, 2015.
[3] J. Molina, X. Zhang, and D. Angland, "On the unsteady motion and stability of a heaving airfoil in ground effect," Acta Mechanica Sinica, vol. 27, no. 2, pp. 164-178, 2011.

[4] W. F. Phillips and D. F. Hunsaker, "Lifting-line predictions for induced drag and lift in ground effect," Journal of Aircraft, vol. 50, no. 4, pp. 1226-1233, 2013.

[5] S. Geisbauer, N. Schade, and S. Enk, "Experimental and numerical investigation of the flow topology during airdrop operations," in Proceedings of the 21st AIAA Aerodynamic Decelerator Systems Technology Conference, pp. 1-11, AIAA, Reston, Va, USA, 2011.

[6] H. Sadat-Hoseini, S. A. Fazelzadeh, A. Rasti, and P. Marzocca, "Final approach and flare control of a flexible aircraft in crosswind landings," Journal of Guidance, Control, and Dynamics, vol. 36, no. 4, pp. 946-957, 2013.

[7] R. Liu, X. X. Sun, W. H. Dong, D. Wang, and Y. Chang, "Dynamics modeling and L1 adaptive control of a transport aircraft for heavyweight airdrop," Mathematical Problems in Engineering, vol. 2015, Article ID 365130, 15 pages, 2015.

[8] O. Härkegård and S. T. Glad, "Resolving actuator redundancyoptimal control vs. control allocation," Automatica, vol. 41, no. 1, pp. 137-144, 2005.

[9] H. Li, Y.-A. Zhang, F.-C. Sun, and W.-H. Li, "Global set-stable nonlinear adaptive dynamic control allocation for aircraft with multiple control effectors," Control and Decision, vol. 28, no. 3, pp. 379-390, 2013.

[10] S. C. Tong and Y. M. Li, "Adaptive fuzzy output feedback tracking backstepping control of strict-feedback nonlinear systems with unknown dead zones," IEEE Transactions on Fuzzy Systems, vol. 20, no. 1, pp. 168-180, 2012.

[11] Y. M. Li, S. C. Tong, and T. S. Li, "Adaptive fuzzy output feedback dynamic surface control of interconnected nonlinear 
pure-feedback systems," IEEE Transactions on Cybernetics, vol. 45, no. 1, pp. 138-149, 2015.

[12] Y. M. Li, S. C. Tong, Y. J. Liu, and T. S. Li, "Adaptive fuzzy robust output feedback control of nonlinear systems with unknown dead zones based on a small-gain approach," IEEE Transactions on Fuzzy Systems, vol. 22, no. 1, pp. 164-176, 2014.

[13] Y. M. Li and S. C. Tong, "Adaptive fuzzy output-feedback control of pure-feedback uncertain nonlinear systems with unknown dead zone," IEEE Transactions on Fuzzy Systems, vol. 22, no. 5, pp. 1341-1347, 2014.

[14] S.-C. Tong, Y.-M. Li, G. Feng, and T.-S. Li, "Observer-based adaptive fuzzy backstepping dynamic surface control for a class of MIMO nonlinear systems," IEEE Transactions on Systems, Man, and Cybernetics, Part B: Cybernetics, vol. 41, no. 4, pp. 1124-1135, 2011.

[15] R. D. Nussbaum, "Some remarks on a conjecture in parameter adaptive control," Systems \& Control Letters, vol. 3, no. 5, pp. 243-246, 1983.

[16] S. S. Ge, F. Hong, and T. H. Lee, "Adaptive neural control of nonlinear time-delay systems with unknown virtual control coefficients," IEEE Transactions on Systems, Man, and Cybernetics, Part B: Cybernetics, vol. 34, no. 1, pp. 499-516, 2004.

[17] Y. T. Wen and X. M. Ren, "Neural networks-based adaptive control for nonlinear time-varying delays systems with unknown control direction," IEEE Transactions on Neural Networks, vol. 22, no. 10, pp. 1599-1612, 2011. 


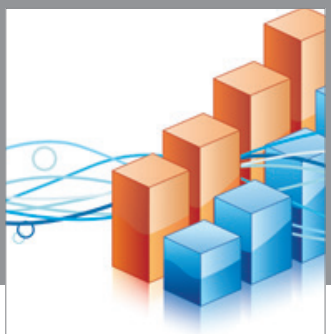

Advances in

Operations Research

vatem alat4

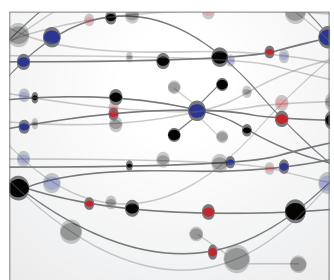

\section{The Scientific} World Journal
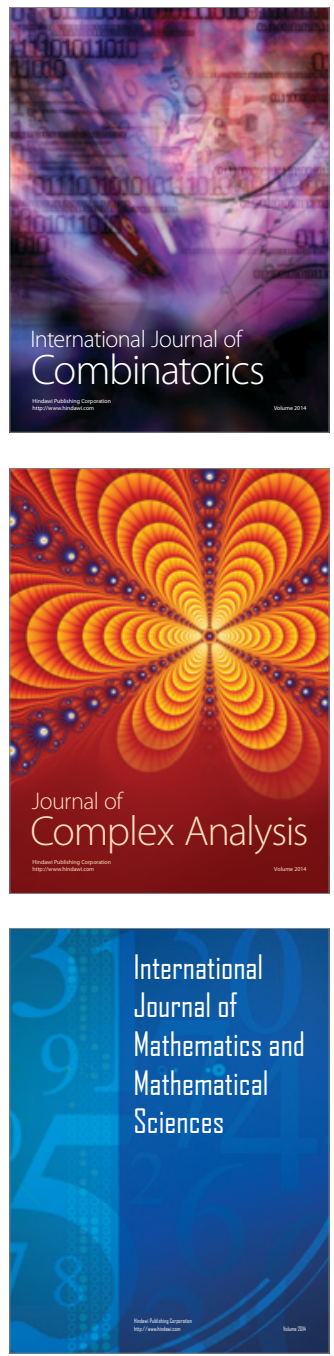
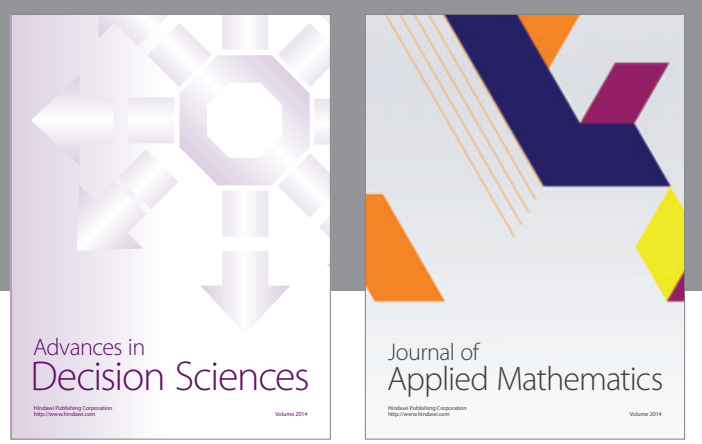

Algebra

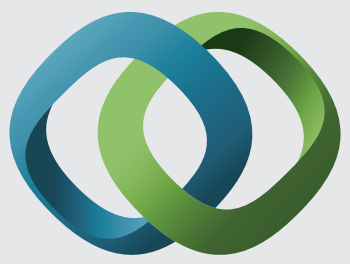

\section{Hindawi}

Submit your manuscripts at

http://www.hindawi.com
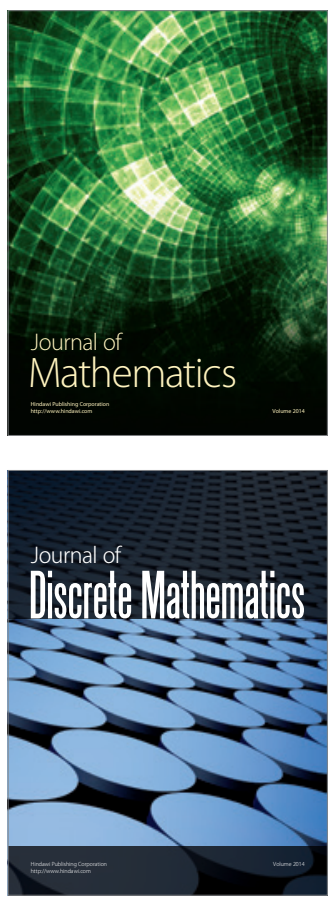

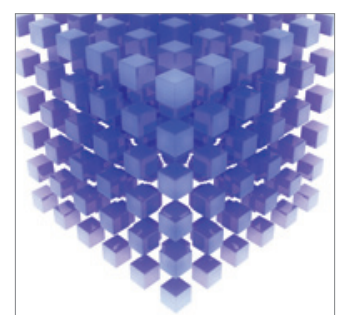

Mathematical Problems in Engineering
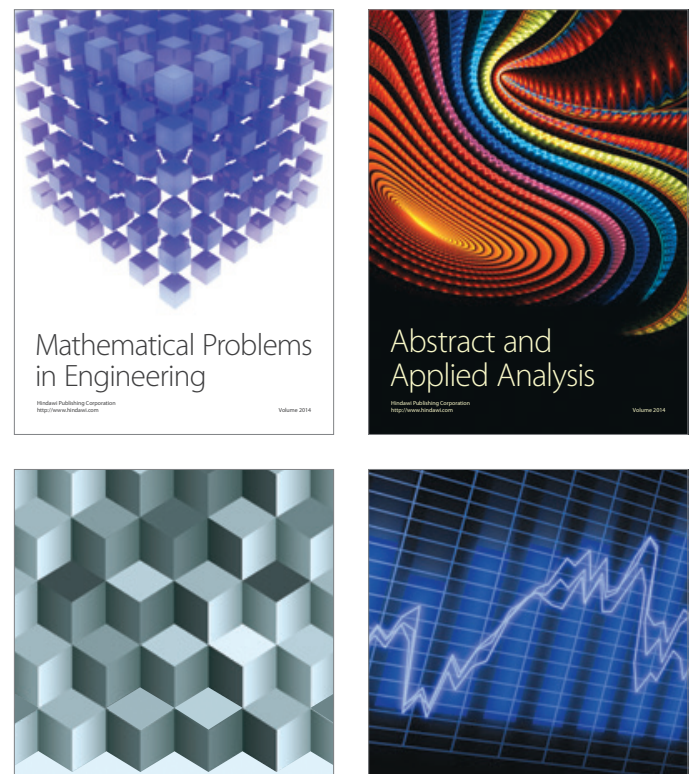

Journal of

Function Spaces

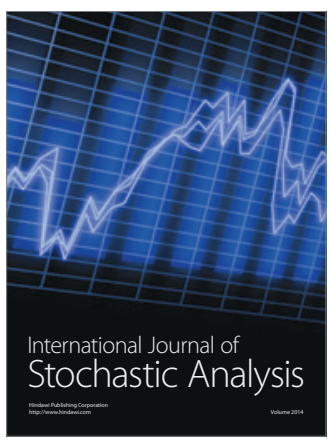

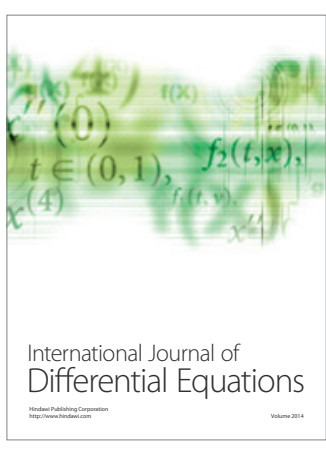
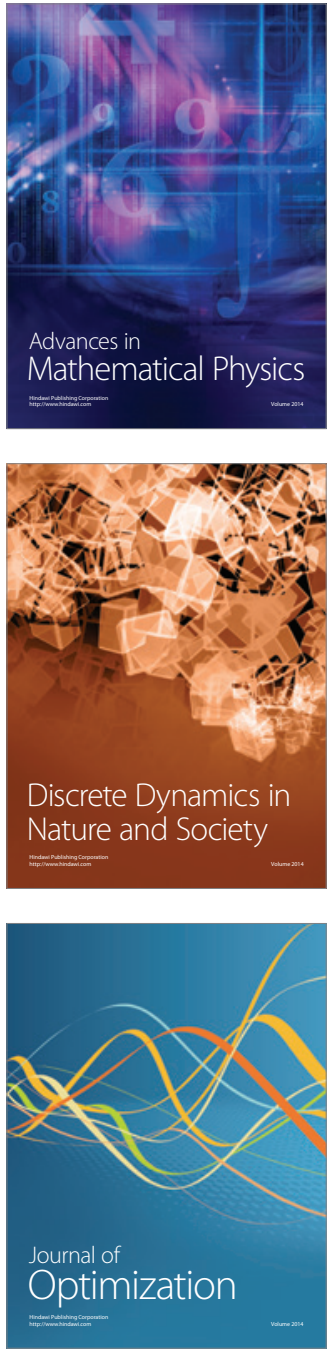